\title{
Monochromatic STEM-EELS for Correlating the Atomic Structure and Optical Properties of Two-Dimensional Materials
}

\author{
Wu Zhou ${ }^{1}$, Niklas Dellby ${ }^{2}$, Leonardo Basile ${ }^{3,4}$, Toshihiro Aoki ${ }^{5}$, Juan Salafranca ${ }^{1,6}$, John Mardinly $^{5}$, Ray \\ Carpenter $^{5}$, Ondrej L. Krivanek ${ }^{2}$, Juan-Carlos Idrobo ${ }^{3}$, Stephen J. Pennycook ${ }^{7}$ \\ ${ }^{1}$ Materials Science and Technology Division, Oak Ridge National Laboratory, Oak Ridge, TN 37831, \\ USA. \\ ${ }^{2}$ Nion Co. Kirkland, Washington, USA. \\ ${ }^{3}$ Center for Nanophase Materials Sciences, Oak Ridge National Laboratory, Oak Ridge, TN 37831 , \\ USA. \\ ${ }^{4}$ Departamento de Física, Escuela Politécnica Nacional, Quito, Ecuador. \\ ${ }^{5}$ LeRoy Eyring Center for Solid State Sciences, Arizona State University, Arizona, USA. \\ ${ }^{6}$ Departamento de Física Aplicada III, Universidad Complutense de Madrid, Spain. \\ ${ }^{7}$ Department of Materials Science and Engineering, University of Tennessee, Knoxville, TN 37996 , \\ USA
}

The introduction of a monochromator can drastically reduce the energy spread of the incident electron beam, and, thus, improve the energy resolution of electron energy loss spectroscopy (EELS). This becomes particularly important for exploring the optical properties of two-dimensional materials using low-loss EELS, where the optical absorption features are typically very weak and could easily be overwhelmed by the tail of the zero-loss peak (ZLP). Furthermore, combined with the imaging power available on an aberration-corrected scanning transmission electron microscope (STEM), simultaneous study and direct correlation of the local atomic structure and optical properties at the nm scale becomes feasible, a power not achievable with optical microscopy and spectroscopy methods.

In this study, we use twisted bilayer graphene (TBG) as a model 2D material system to demonstrate the simultaneous study of the local atomic structure and optical properties with monochromatic STEM-EELS. The experiment was performed on an aberration-corrected Nion UltraSTEM, equipped with a cold field emission electron source and a newly designed monochromator [1], operated at $60 \mathrm{kV}$ accelerating voltage.

Figure 1 compares the ZLP acquired with and without the monochromator. The monochromator improves the energy resolution from $300 \mathrm{meV}$ to $60 \mathrm{meV}$ with 1 minute acquisition time, while $12 \mathrm{meV}$ energy resolution is achievable with ms acquisition [1]. Importantly, even though the energy resolution is improved only by a factor of 5, the relative intensity of the ZLP tail below $1 \mathrm{eV}$ drastically decreases by more than two orders of magnitude when the monochromator is switched on, as shown in the inset of Figure 1. This suggests that for 2D materials, where plural scattering is negligible, the Nion monochromator can increase the information transfer capability by a factor of 100 for optical response in the near- and mid-infrared regime. This is further demonstrated in Figure 2, where the low-loss EEL spectra for a few TBG samples acquired with and without the monochromator are presented. The spectra clearly reveal the presence of additional optical absorption peaks (highlighted), which shift consistently as a function of the twisted angle in TBG and can be well described by tight-binding calculations. Noticeably, without the monochromator, this additional optical feature can only be identified in the visible and ultraviolet regime, while monochromatic EELS provides access to this new feature all the way down to the mid-infrared regime, superior to previous optical studies [2]. Other examples on h-BN, 
as well as important technical details and probe-position-dependent monochromatic EELS results, will also be presented [3].

\section{References:}

[1] O L Krivanek et al, Microscopy 62 (2013) 3-21.

[2] R W Havener et al, arXiv:1309.2011 (2013).

[3] This research was supported by a Wigner Fellowship through the Laboratory Directed Research and Development Program of Oak Ridge National Laboratory (ORNL), managed by UT-Battelle, LLC, for the U.S. DOE (WZ), the National Secretariat of Higher Education, Science, Technology and Innovation of Ecuador (SENESCYT) (LB), ERC starting Investigator Award, grant \#239739 STEMOX, and Juan de la Cierva program JCI-2011-09428 (MICINN-Spain) (JS), the Center for Nanophase Materials Sciences (CNMS), which is sponsored at ORNL by the Scientific User Facilities Division, Office of Basic Energy Sciences, U.S. DOE (JCI), and by the Office of Basic Energy Sciences, Materials Sciences and Engineering Division, U.S. DOE (SJP).

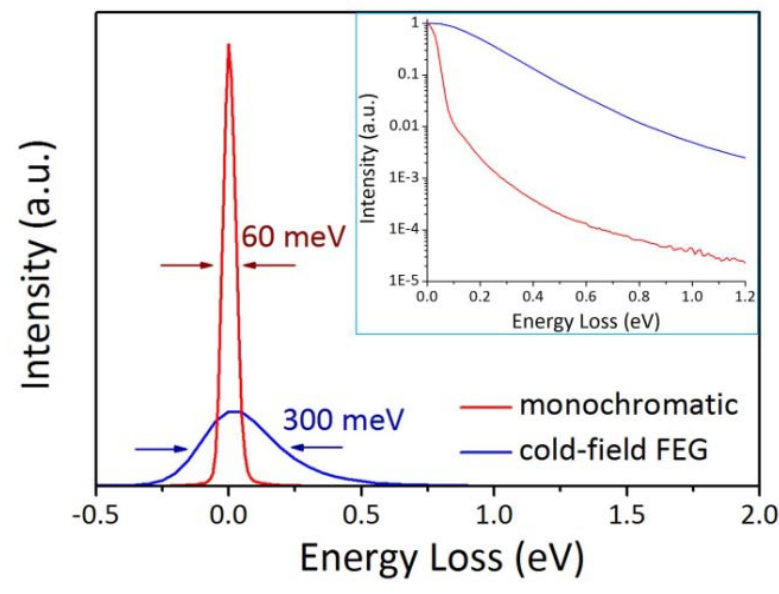

Figure 1. Comparison of the ZLP taken on the Nion UltraSTEM with and without the monochromator. The main figure is normalized to the integrated intensity, while the inset is normalized to the maximum intensitv of the ZLP.
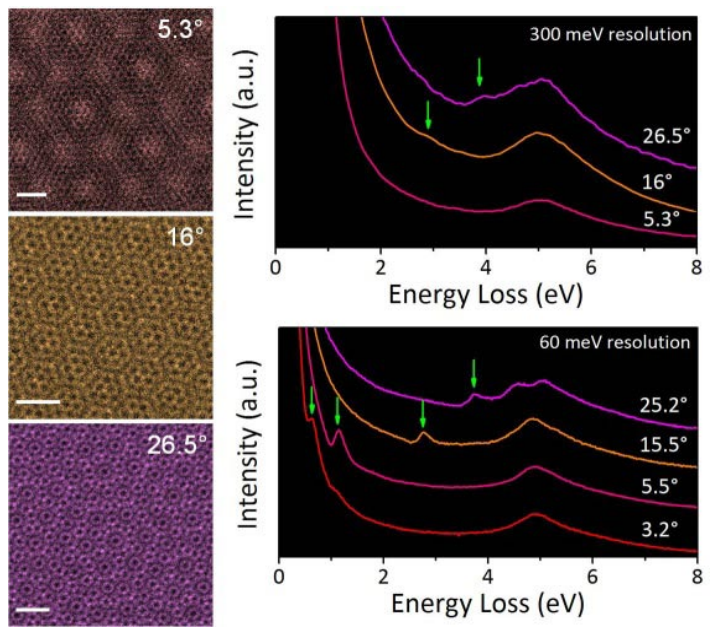

Figure 2. (Left) Experimental ADF images of twisted bilayer graphene with different misorientation angles. Scale bars: $1 \mathrm{~nm}$. (Right) Low-loss EEL spectra of the TBG acquired using the Nion UltraSTEM with and without the monochromator. The misorientation angles are labeled on each spectrum. 